\title{
Quantum simulation of the Anderson Hamiltonian with an array of coupled nanoresonators: delocalization and thermalization effects
}

\author{
John Lozada-Vera', Alejandro Carrillo', Olimpio P de Sá Neto², Jalil K Moqadam¹, Matthew D LaHaye and
} Marcos $C$ de Oliveira ${ }^{*}$

\author{
"Correspondence: \\ marcos@ifi.unicamp.br \\ "Instituto de Física "Gleb Wataghin", \\ Universidade Estadual de Campinas, \\ Campinas, SP 13083-970, Brazil \\ Full list of author information is \\ available at the end of the article
}

\begin{abstract}
The possibility of using nanoelectromechanical systems as a simulation tool for quantum many-body effects is explored. It is demonstrated that an array of electrostatically coupled nanoresonators can effectively simulate the Bose-Hubbard model without interactions, corresponding in the single-phonon regime to the Anderson tight-binding model. Employing a density matrix formalism for the system coupled to a bosonic thermal bath, we study the interplay between disorder and thermalization, focusing on the delocalization process. It is found that the phonon population remains localized for a long time at low enough temperatures; with increasing temperatures the localization is rapidly lost due to thermal pumping of excitations into the array, producing in the equilibrium a fully thermalized system. Finally, we consider a possible experimental design to measure the phonon population in the array by means of a superconducting transmon qubit coupled to individual nanoresonators. We also consider the possibility of using the proposed quantum simulator for realizing continuous-time quantum walks.
\end{abstract}

Keywords: quantum simulators; nanoelectromechanical system; Anderson localization

\section{Introduction}

The achievement of the ground state of mechanical motion using nanoelectromechanical systems (NEMS), as demonstrated recently in remarkable experiments [1-6], opens up a new path for studying quantum behavior in macroscopic systems. Having been able also to coherently control and cleverly measure the state of the mechanical resonator [1,7-11], an immediate possibility to explore is the use of the NEMS as building blocks for fabricating analog quantum simulators to reproduce many-body quantum physics [12, 13]. Analog quantum simulators are dedicated and controllable devices, which can imitate (within some accuracy) the evolution of certain types of Hamiltonians. Various quantum systems have already been investigated for quantum simulation. Previously, quantum simulators were experimentally implemented using ultracold quantum gases [14], trapped ions [15], photonic quantum systems [16] and superconducting circuits [17, 18]. Recently, an array

(c) 2016 Lozada-Vera et al. This article is distributed under the terms of the Creative Commons Attribution 4.0 International License (http://creativecommons.org/licenses/by/4.0/), which permits unrestricted use, distribution, and reproduction in any medium, provided you give appropriate credit to the original author(s) and the source, provide a link to the Creative Commons license, and indicate if changes were made. 
of optomechanical resonators has been suggested for simulating many-body nonlinear driven dissipative quantum dynamics [19].

One particularly important phenomena that emerges due to the wave-like nature of matter at the quantum regime is Anderson localization [20-22], a phenomena in which waves fail to propagate in disordered media due to interference. Anderson localization has been observed with many experimental setups, including microwaves [23-25], light waves [26-32], ultrasound [33,34] and matter waves [35-40]. Given the ability to achieve the quantum regime in mechanical resonators, it would be extremely appealing to observe mechanical localization in an array of NEMS, where thermal effects become relevant. Furthermore, arrays of NEMS could be used to simulate continuous-time quantum walk (CTQW) dynamics [41]. Quantum walks are the quantum version of random walks that have a crucial role in designing efficient quantum algorithms that outperform classical algorithms [42]. Implementation of CTQW has been already realized in a four-site circle using a two-qubit nuclear magnetic resonance quantum computer [43] and in a waveguide array [44]. A NEMS-based quantum simulator would provide a means to implement CTQW with phonons, opening up new opportunities for quantum algorithms and universal quantum computation [45].

In this paper, we propose a 1D array of coupled nanomechanical resonators for simulating the Anderson Hamiltonian, namely, a discrete tight-binding model without on-site interactions. The coupling between resonators is electrostatic (capacitive coupling), but could also be mechanic (elastic coupling) in order to be improved. The disorder can be induced in a controlled and predetermined manner through the appropriate variation in the design and nanofabrication of the geometrical dimensions of the nanoresonators in the array [46]. A qubit coupled to the chain can be used as a device for both, initializing and measuring the occupation probabilities of excitations. We also discuss the physical implementations of CTQWs using the proposed quantum simulator.

\section{Model}

An array of $N$ capacitively coupled electromechanical resonators, as depicted in Figure 1 (upper panel), can be described by the Hamiltonian

$$
\mathcal{H}=\sum_{j=1}^{N}\left(\frac{p_{j}^{2}}{2 m_{j}}+\frac{1}{2} m_{j} v_{j}^{2} x_{j}^{2}\right)+\sum_{j=1}^{N-1} U_{j},
$$

where $x_{j}, p_{j}, m_{j}$ and $v_{j}$ are the displacement from the equilibrium position, momentum, mass and frequency, respectively, associated with a single mechanical mode of each resonator. $U_{j}$ is the electrostatic interaction energy between the pair of nearest-neighbor resonators $j$ and $j+1$. The interaction between a resonator and its second nearest neighbor is small enough to be disregarded. Using a simple parallel plate capacitor model [see Figure 1 (coupling)], the energy $U_{j}$ in Eq. (1) can be written as

$$
U_{j}=\frac{1}{2} C_{j} \Delta V_{j}^{2}=\frac{1}{2} \frac{\epsilon_{0} A \Delta V_{j}^{2}}{d+\left[x_{j}(t)-x_{j+1}(t)\right]},
$$

where $C_{j}$ and $\Delta V_{j}=\left|V_{j+1}-V_{j}\right|$ are the capacitance and the voltage difference between the resonators $j$ and $j+1$, respectively. The capacitance $C_{j}$ is expressed in terms of the 
Figure 1 A chain of electrostatically coupled mechanical resonators. Every single resonator is charged by an individual source, to induce electric potential diferences, and is considered as non-ideal by being coupled to individual thermal reservoirs. Imperfections in the resonators fabrication naturally introduce a disorder in their respective principal oscillation mode, hence, Anderson localization of phonon excitations can be observed for a sufficiently large array and sufficiently low temperature. Depicted quantities are discussed within the text.

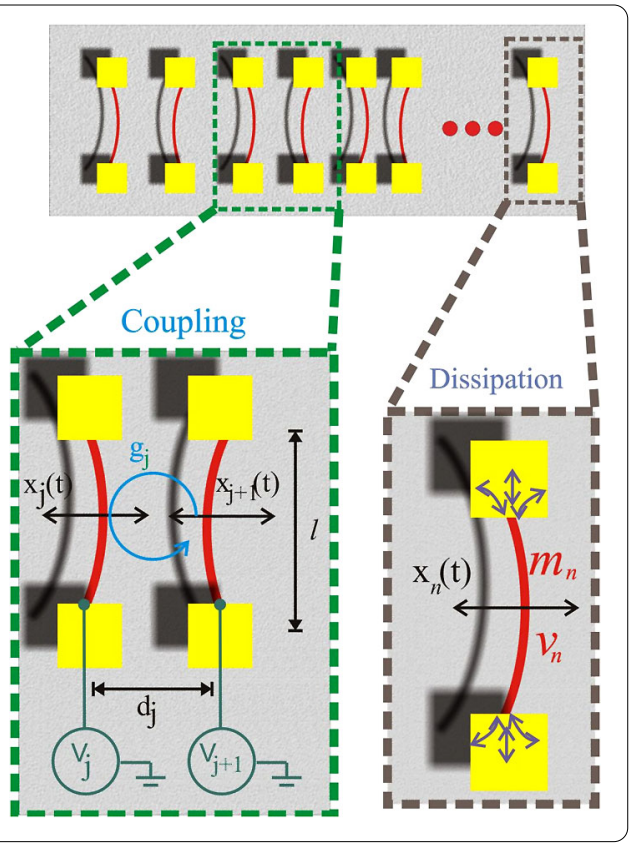

vacuum permittivity $\epsilon_{0}$, the resonator area $A$ and the equilibrium center-of-mass separation $d$. Note that the inevitable disorder appearing in $d$ is here neglected because its effect would be to introduce a disorder in the coupling energies, which are too small to be taken into account. The oscillation amplitudes are close to the zero point fluctuations, hence, $\left|\delta x_{j}(t)\right|=\left|x_{j}(t)-x_{j+1}(t)\right| \ll d$ and the electrostatic potential (2) can be expanded in powers of $\delta x_{j}(t) / d$, which up to the second order gives

$$
U_{j} \simeq \frac{\epsilon_{0} A \Delta V_{j}^{2}}{2 d}\left[1-\frac{\delta x_{j}(t)}{d}+\frac{\left[\delta x_{j}(t)\right]^{2}}{2 d^{2}}\right] .
$$

The linear terms will cancel out in the summation of $U_{j}$, and only remaining linear terms are $\left(x_{1}(t)-x_{N}(t)\right) / d$, which nonetheless will also be negligible in comparison to the remaining second order terms, due to the rapid oscillation of $x_{j}(t)$. We now quantize the system, by replacing the classical variables with the operators written in terms of the annihilation and creation operators of the resonator excitation, $a_{j}$ and $a_{j}^{\dagger}$. In the interaction picture, the position and momentum operators are given by $\hat{x}_{j}(t)=\sqrt{\hbar /\left(2 m_{j} v_{j}\right)}\left(a_{j}^{\dagger} e^{i v_{j} t}+a_{j} e^{-i v_{j} t}\right)$ and $\hat{p}_{j}=i \sqrt{\hbar m_{j} v_{j} / 2}\left(a_{j}^{\dagger} e^{i v_{j} t}-a_{j} e^{-i v_{j} t}\right)$, respectively. Substituting them in Eq. (3), the rapidly oscillating terms vanish in the rotating wave approximation (RWA) and Hamiltonian (1) is converted to

$$
\mathcal{H}=\sum_{j=1}^{N} \hbar \omega_{j} a_{j}^{\dagger} a_{j}-\sum_{j=1}^{N-1} \hbar g_{j}\left(a_{j}^{\dagger} a_{j+1}+a_{j} a_{j+1}^{\dagger}\right),
$$

where $g_{j}=\epsilon_{0} A \Delta V_{j}^{2} x_{j}^{\mathrm{zpf}} x_{j+1}^{\mathrm{zpf}} / 2 d^{3}$ is the tunnelling energy, $x_{j}^{\mathrm{zpf}}=\sqrt{\hbar /\left(2 m_{j} v_{j}\right)}$ is the zero point fluctuation of the resonator $j$ and $\omega_{j}=v_{j}+\frac{\epsilon_{0} A \Delta V_{j}^{2}}{2 d^{3} m_{j} v_{j}}$ is the rescaled mode frequency. $\omega_{j}$ is randomly distributed over the range $[\bar{\omega}-\Delta, \bar{\omega}+\Delta]$, where $\bar{\omega}$ is the average frequency of the resonators and $\Delta$ is called the disorder intensity. We assume that the difference between any pair of disordered mode frequencies is small, hence the resonance condition is almost 
satisfied. In other words $\Delta \ll \bar{\omega}$. In fact for all simulations considered here we have assumed $\Delta \leq 10^{-2} \bar{\omega}$. Moreover in any practical situation $\bar{\omega} \gg g_{j}$, but $\Delta \geq g_{j}$ (see Section 5 for discussion of engineering the system parameters, and Table 1 for experimental values), and therefore the validity of the RWA is always assured. Note that the coupling energies $g_{j}$ depend on several experimental parameters related to the resonator fabrication such as mass, frequency and geometry, as well as the chain parameters such as the distance between the resonators and the potential difference. Therefore they could also be random variables that would define an off-diagonal disorder. However, since $\omega_{j} \gg g_{j}$, the disorder in $g_{j}$ is negligible in comparison to the diagonal one introduced by $\Delta$. To simplify the calculations we fix all the tunneling energies to $g_{i}=J$.

The Hamiltonian (4) is the Bose-Hubbard Hamiltonian without inter-mode interactions, and a diagonal disorder in the on-site energies $\omega_{j}$. When the total number of phonons is restricted to 1, this Hamiltonian reduces to the Anderson tight-binding Hamiltonian (see below).

\section{Closed system: Anderson localization}

Localization, from a general point of view, is a mesoscopic phenomena displayed by waves as they propagate through a disordered medium. It is built upon the interference of the many randomly scattered waves, which at sufficiently large distances, collude to produce a suppression in the amplitude of propagation. This behavior is characterized by the exponential decay of the wave-function, with a decay length $\xi$, known as the localization length. The celebrated scaling theory of localization [47] describes how the transition between the different diffusion regimes depend upon the size $L$, and dimension $D$, of the system, independent of the microscopic intricacies of the disorder. According to the theory, depending on $L$, three different transport regimes can be recognized: ballistic $(\xi \gg L)$, where the size of the system is too small and scattering events are rare; diffusive $(\xi \lesssim L)$, where some weak localization effects may take place; and strong localization $(\xi \ll L)$, for large systems [48]. Of course, the detailed form of the localization depends on the type of disorder potential considered and its energy spectrum.

In the Anderson model (which is the one of interest in this paper), disorder is modeled by a $\delta$-correlated potential $V$, consisting of a series of spatially uncorrelated barriers, ${ }^{\text {a }}$ with a finite maximum amplitude intensity $\Delta$, called disorder strength. One can as well picture a periodic lattice with randomly shuffled on-site energies. Strictly speaking, a proper phase transition from extended to localized states only exists for $D=3$, at some critical disorder intensity or, interchangeably, some critical energy known as the mobility edge. For $D<2$ it can be shown that all states are localized, but the localization length for $D=2$ can easily exceed the size of the system for weak disorder or high enough particle energies, thus admitting the existence of a diffusive transport regime. In contrast, for $D=1$, (almost ${ }^{\mathrm{b}}$ ) all single-particle eigenstates are localized even for a vanishingly small $\Delta$ and there is no phase transition. ${ }^{\mathrm{c}}$

For the description of the linear chain of resonators, we can disregard the free propagation between lattice sites, focusing only on the occupation amplitudes of each site. ${ }^{\mathrm{d}}$ In the single-phonon situation, Hamiltonian (4) turns into $(\hbar=1)$ :

$$
\mathcal{H}_{A}=\sum_{j=1}^{N} \omega_{j}|j\rangle\langle j|+J(|j\rangle\langle j+1|+| j+1\rangle\langle j|),
$$


Figure 2 Population second moment. Average over 500 disorder realizations of the relative second moment in the population distribution $\Delta n / n_{\text {av }}$ for the ground state of the Anderson Hamiltonian, as a function of the system size $N$ and for different disorder intensities (from top to bottom $\Delta / J=2,5,10,15,20$ ) showing the convergence of the dispersion of the population as the number of sites is increased.

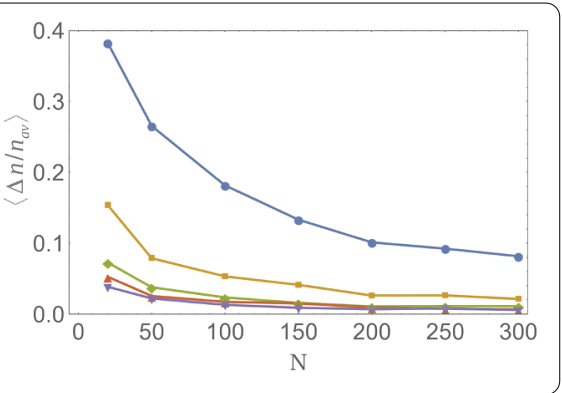

where the states $|j\rangle$ are the occupation amplitudes of each resonator, $J$ is the coupling rate between neighbouring sites, and the set of $\omega_{j}$ are assumed to follow a uniform random distribution around the mean value $\omega$. For this model, numerical simulations are easy to perform, for example using closed boundary conditions at both edges of the chain, and diagonalizing Hamiltonian (5) directly, varying the total number of sites $N$ and disorder intensity $\Delta$, for many realizations of the disordered potential. ${ }^{\mathrm{e}}$ To set the stage for further discussions, we start in Figure 2 by plotting the behavior of the averaged relative dispersion in the site population for the ground state of the resonators $\left|\psi^{0}\right\rangle$, given by $\left\langle\left\langle\Delta n / n_{\mathrm{av}}\right\rangle\right\rangle=$ $\left\langle\left\langle\left[\sum_{j}^{N} j^{2}\left|\psi_{j}^{(0)}\right|^{2}-n_{\mathrm{av}}^{2}\right]^{1 / 2} / n_{\mathrm{av}}\right\rangle\right\rangle$, as a function of the system size ( $N$ ranging between 1 to 300 sites) and for different disorder strengths $(\Delta / J=2,5,10,15,20)$. Here $n_{\mathrm{av}}=\sum_{j}^{N} j\left|\psi_{j}^{(0)}\right|^{2}$, is simply the mean value of the site population, and $\psi_{j}^{(0)}=\left\langle j \mid \psi^{0}\right\rangle$ are the different population amplitudes for the ground-state; the double angular brackets denote the average over the disorder realizations. It can be noted the sensibility of the model for small sizes, since although the eigenstates are always localized, the relative dispersion in population sites only converges for a large enough system, a situation that is more relevant as the disorder gets weaker as can be seen comparing the different curves for the region $N \lesssim 50$. This dependence on the system size is clear in the curve in Figure 2 for $\Delta / J=2$. It would be required a much larger system for the curve to converge closer to the other ones.

The typical exponential profile for the population density is plotted in Figure 3(a) for $\Delta / J=15$. A single excitation in the central site for a chain of 51 resonators is assumed as the initial condition. In a Bose-Einstein Condensate (BEC), the measured quantity of interest is the density of states at a given position in the lattice, namely, $|\psi(x)|^{2}[35-40]$. In our simulator, the quantity to be measured is the population of the first excited state of a given resonator $j$, which is $\rho_{\left|\omega_{j}\right\rangle\left\langle\omega_{j}\right|}$. Given the discrete nature of our system, we cannot expect a smooth Gaussian-to-exponential transition in the population profile. However, we see that due to the presence of disorder in the diagonal terms of the Hamiltonian, $\omega_{j}$ in Eq. (4), the spatial distribution of the excitations present in the chain remain always close to the initial spatial distribution. Note that in Figure 3 the initial nonstationary regime is not shown. This short time which is disregarded corresponds to the transitory path to equilibrium.

It is interesting to explore the physics that is difficult to be included in the Anderson model, and which is nonetheless present in a real implementation of the system. Specifically, the influence of thermal effects due to a surrounding reservoir for the resonators is considered in the following. 

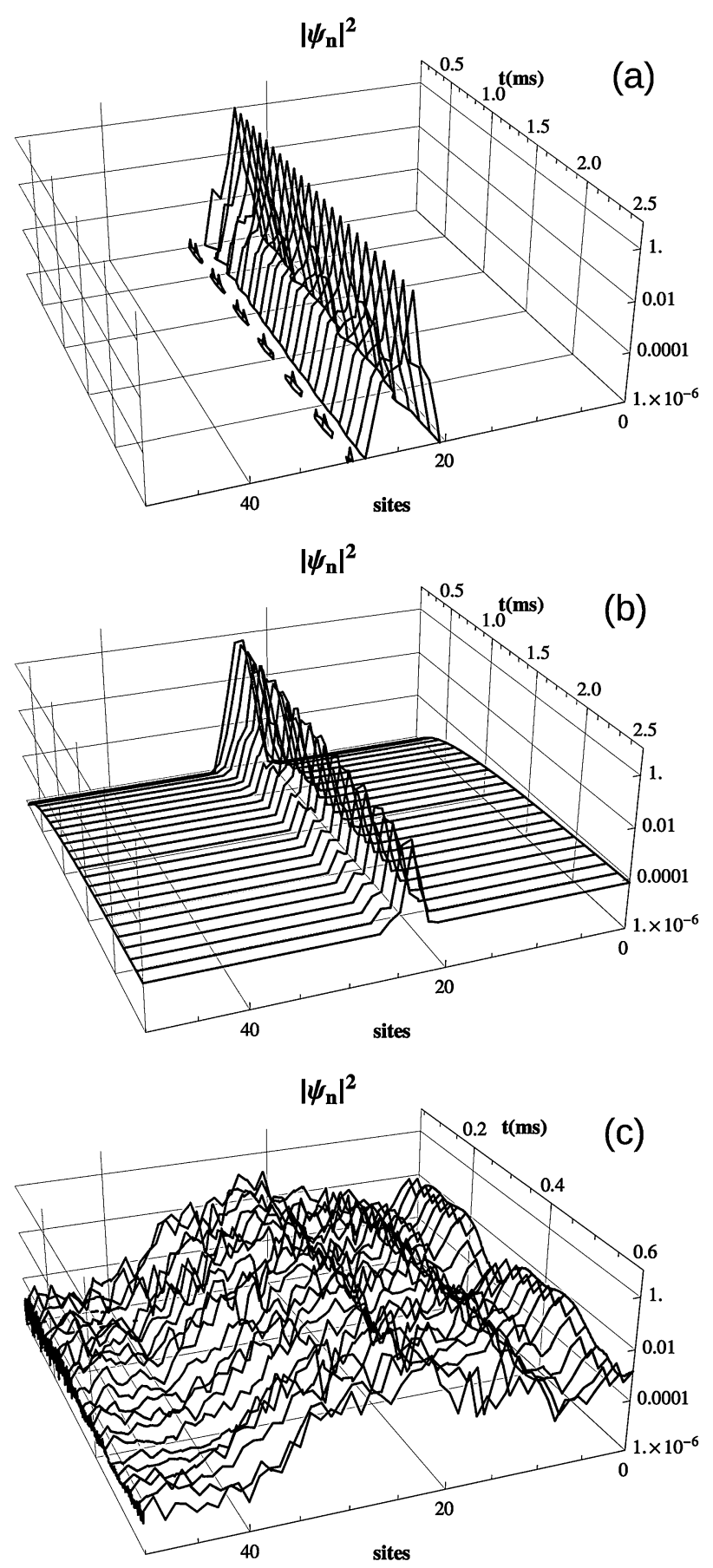

Figure 3 Density profiles. Density profiles of the phonon population for a chain of 51 nanomechanical resonators. (a) Depicts the situation for a closed system with $\Delta / J=15$, while (b) and (c) for open systems affected by thermal reservoirs with $\bar{n}=10^{-2}$ and $\gamma=1 \mathrm{MHz} \cdot \ln$ (b) $\Delta / J=15$; in (c) $\Delta / \mathrm{J}=2$.

\section{Open system}

Here, we write a master equation for the Anderson Hamiltonian (4) to describe the effects of the environment on the chain and consequently to investigate the corresponding influence on the localization of the states. In this way, we need to take into account phonons 
and weak electromagnetic fields that are surrounding the nanoresonators. Assuming that each resonator in the chain is coupled to a bosonic bath, the corresponding interaction Hamiltonian takes the form

$$
\mathcal{H}_{\mathrm{I}}=-\sum_{j, l} \Gamma_{j l}\left(a_{j}^{\dagger} b_{j, l}+a_{j} b_{j, l}^{\dagger}\right)
$$

where $b_{j, l}$ and $b_{j, l}^{\dagger}$ are the $j$ th resonator bath annihilation and creation operators and $\Gamma_{j l}$ is the coupling between resonator-bath modes. In order to derive the master equation it is supposed that each resonator is weakly coupled to its respective bath, and that all bosonic baths are in thermal equilibrium, with mean thermal phonon number $\bar{n}$. The master equation describing the dynamics of the chain is then

$$
\begin{aligned}
\dot{\rho}_{S}(t)= & -\frac{i}{\hbar}\left[\mathcal{H}, \rho_{S}(t)\right]+\sum_{j} \frac{\gamma_{j}}{2}\left[\bar{n}\left(2 a_{j}^{\dagger} \rho_{S}(t) a_{j}-a_{j} a_{j}^{\dagger} \rho_{S}(t)-\rho_{S}(t) a_{j} a_{j}^{\dagger}\right)\right. \\
& \left.+(\bar{n}+1)\left(2 a_{j} \rho_{S}(t) a_{j}^{\dagger}-\rho_{S}(t) a_{j}^{\dagger} a_{j}-a_{j}^{\dagger} a_{j} \rho_{S}(t)\right)\right],
\end{aligned}
$$

where $\rho_{S}$ is the system density operator, $\gamma_{j}$ is the effective resonator-bath interaction strength and $\bar{n}$ is the bath mean thermal phonon number, which is specified by using the Bose-Einstein statistics. We have considered $\gamma_{j}=\gamma=1 \mathrm{MHz}$ for all simulations in this work. Remark that since we are interested in considering the Anderson model for a single phonon population in the chain we must keep the temperature reasonably low. Considering actual temperatures reached in experiments for mechanical resonators in the quantum regime, this corresponds to very low $\bar{n}$. We have considered $\bar{n}$ varying from $10^{-4}$ to $10^{-1}[1]$, which nonetheless is sufficient to see a disturbance in the localization mechanism, without compromising our numerical simulation.

The effect of the reservoir on the system include loss of excitations due to imperfections in the medium. It simply means that the single phonons in the chain, eventually would leak into the medium. This phenomena can be understood like an incoherent spontaneous emission. The expected behavior of a system subject to an amplitude damping channel is the escape of all excitations in the chain. However, while such decoherence process is taking effect, we can argue the existence of enough quantum correlations as to assure the existence of localization phenomena. Working in a weak resonator-bath interaction regime (something like three orders of magnitude less than the resonator-resonator interaction), the correlations remain in the system until the leaking process has taken away all measurable probability of excitations. We can clearly see this behavior in Figure 3(b) for $\gamma=1 \mathrm{MHz}, \bar{n}=10^{-2}$, and normalized disorder $\Delta / J=15$. Similarly to Figure 3(a) a single excitation in the central resonator is taken as the initial condition. We see after equilibration a behavior which similar to he one characterising localization in Figure 3(a) but for a increasing uniform thermal excitation base. In Figure 3(c) the same initial condition and dynamics is employed but for a smaller disorder $\Delta / J=2$. Now we see a typical thermal behavior of a non-localized density profile. Remark that for $\gamma=1 \mathrm{MHz}$ there is enough time for equilibration.

In Figure 4 it is depicted more clearly the effect of the thermal excitation over the phonon localization. There it is plotted the time evolution of the resonators phonon population 
Figure 4 Phonon population dispersion. Time evolution of the resonators phonon population dispersion for a chain of 51 nanomechanical resonators. $\langle\langle\Delta n\rangle\rangle$, as a function of the thermal phonon number, $\bar{n}$, for (a) $\Delta / J=2$; and (b) $\Delta / J=15$. The higher $\bar{n}$ gets, the stronger will be the delocalization.

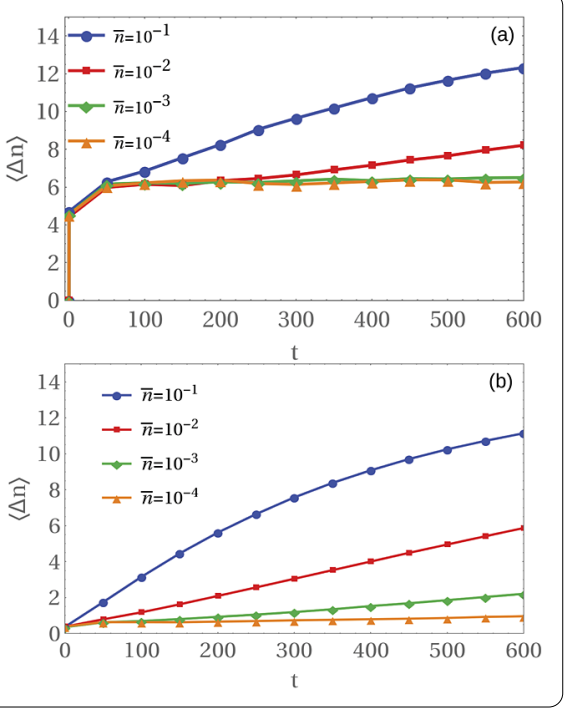

dispersion, $\langle\langle\Delta n\rangle\rangle$, as a function of the thermal phonon number, $\bar{n}$, for two different disorder strengths: 4 (a) $\Delta / J=2$ and 4 (b) $\Delta / J=15$, and for a single excitation in the central resonator as initial condition. It can be seen that the higher the temperature (or $\bar{n}$ ) gets, the stronger will be the delocalizing effect as expected, very much independent of the disorder intensity. However for the lower temperatures (green and orange curves), the phonon population remains localized around the initial excited resonator, even in the presence of leaking in the total population of excitations due to dissipative effects. Furthermore, for the higher temperatures (blue and red), the localization is lost due to thermal pumping of excitations into the array, producing a fully thermalized state.

With an initial condition available experimentally, namely, exciting a central nanomechanical resonator in the chain (see Section 5), the fast dynamics creates correlations in nearby resonators. These correlations imply long-time entanglement between the resonators, which in turn give us the possibility to maintain localization until thermalization is reached. To investigate the correlations in a finite chain of nanoresonators we consider the concurrence which is a bipartite entanglement measure and defined as [49]:

$$
C(\rho)=\max \left\{0, e_{1}-e_{2}-e_{3}-e_{4}\right\}
$$

where $e_{i}$ are the square roots of the eigenvalues in decreasing order of the positive definite matrix $\rho \tilde{\rho}$, where

$$
\tilde{\rho}=\left(\sigma_{y} \otimes \sigma_{y}\right) \rho^{*}\left(\sigma_{y} \otimes \sigma_{y}\right),
$$

in which $\rho^{*}$ is the complex conjugate of the density matrix $\rho$ and $\sigma_{y}$ is the $Y$ Pauli matrix. Concurrence belongs to the interval $[0,1]$ where $C(\rho)=0,1$ corresponds to separable states and maximally entangled states, respectively. we measure the concurrence between the initially excited nanomechanical resonator (at the centre of the chain) and each of the other nanomechanical resonators under different conditions of the disorder and coupling with the environment. The results are shown in Figures 5 and 6.

Figures 5 and 6 show that the behavior of the entanglement is consistent with that of the population probability distribution from Figure 3. The concurrence and the population 


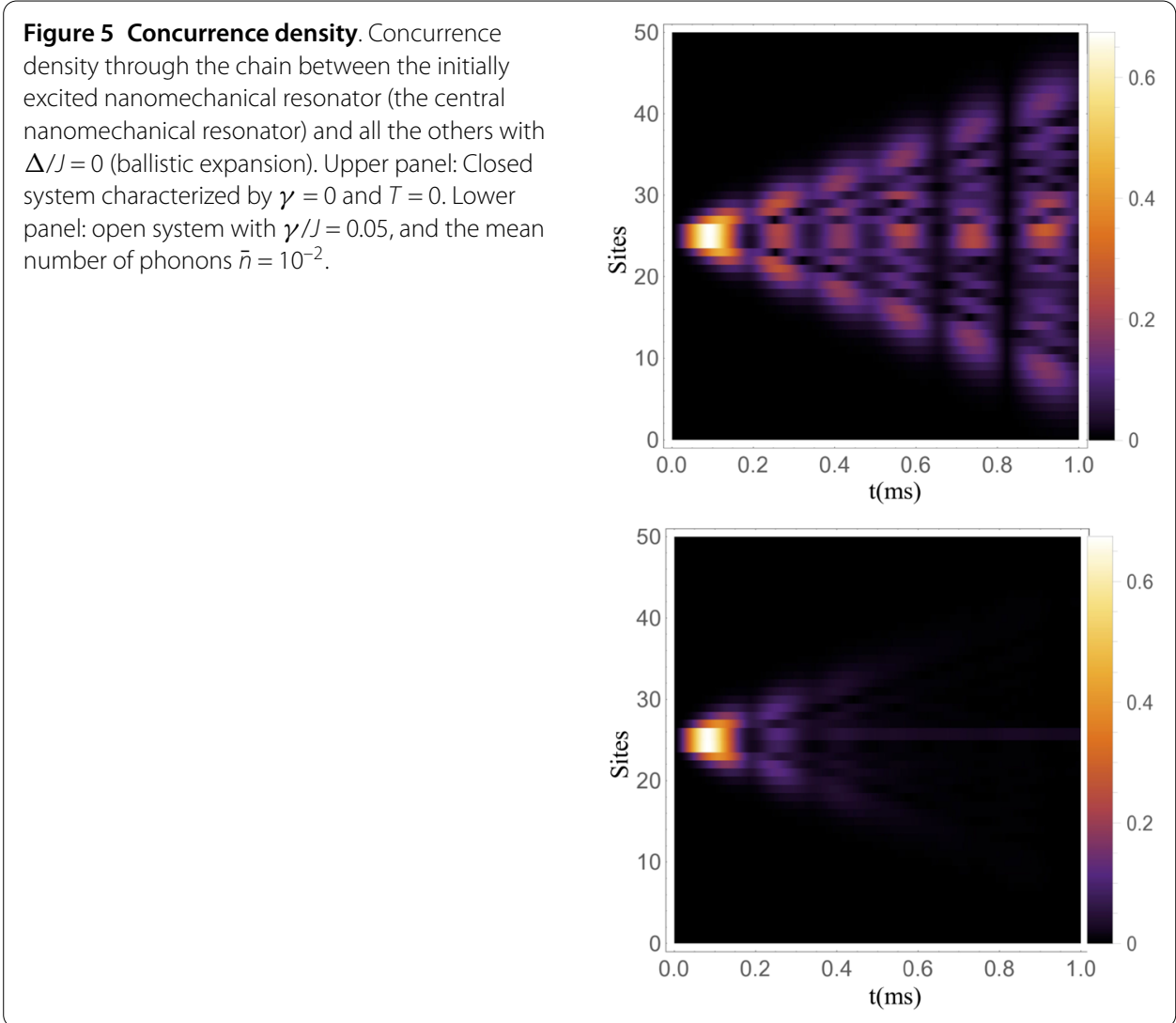

both spread through the available sites or become localized due to the disorder. The presence of an environment does not introduce new dynamics. The only effect of the environment, as expected, is to destroy quantum coherences with a rate proportional to $\exp (-\gamma t)$, regardless of the presence of localization. The figures also show the sudden death of the entanglement [50] in which the concurrence between the central nanomechanical resonator and all the others destroys periodically.

The inclusion of thermal effects does not immediately change the main feature of the localization (Figure 6 (bottom)). The system is quickly localized as expected from the Anderson-like Hamiltonian with disordered diagonal terms. With dissipation, localization is still a quantum effect in the sense that correlations between the resonators remain until the dissipation takes away all possible dynamics. As time goes on, depending on the temperature, all the states become thermalized. Even for very low temperatures, before all excitations leave the chain, the equilibrium state will be a thermalized state. However, the thermal relaxation rate is slow enough that localized phonon populations could still be measured prior to decaying.

Continuous-Time Quantum Walk. The Anderson Hamiltonian given in Eq. (4) also generates CTQW dynamics. For a small disorder, an initial phonon injected in the central nanoresonator spreads ballistically and the standard deviation of the corresponding probability distribution over the chain increases linearly with time. The measurement method which is described in the following section can be used to reconstruct the probability distribution, shown in Figure 7. It is also possible to inject two or more phonons in the chain 
Figure 6 Concurrence density. Concurrence density through the chain between the initially excited nanomechanical resonator (the central nanomechanical resonator) and all the others with $\Delta / J=10$, that is the localized system. Upper panel: Closed system characterized by $\gamma=0$ and $T=0$. Lower panel: open system with $\gamma / \mathrm{J}=0.05$, and a mean number of phonons $\bar{n}=10^{-2}$.
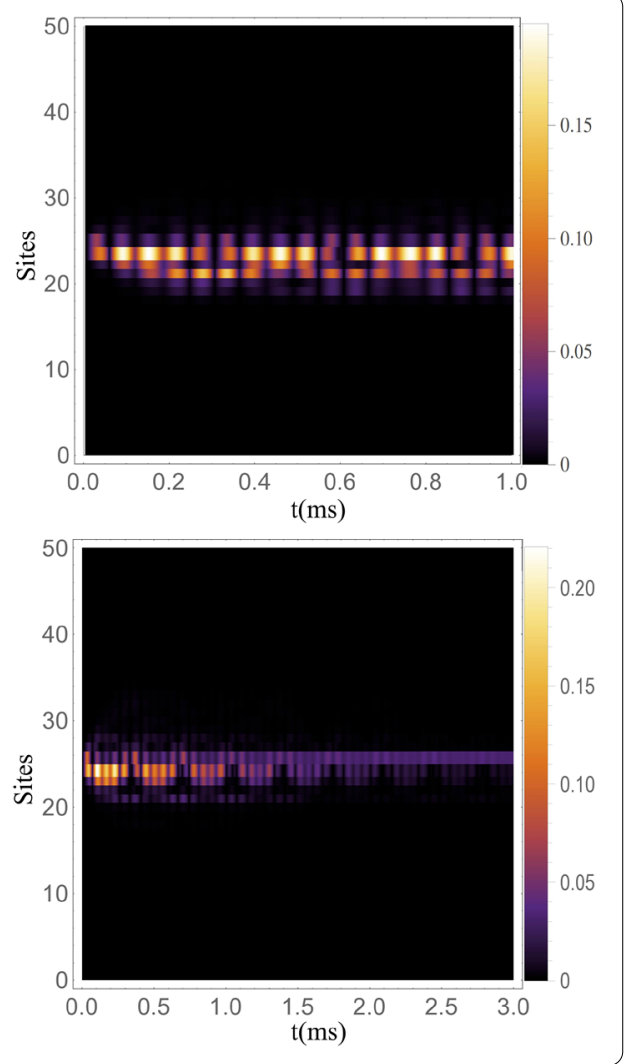

to investigate the multi-particle quantum walks. That, specifically, provides a means for simulating bosonic particles and is going to be addressed elsewhere.

\section{Measurement}

Quantum measurement is a crucial step in quantum simulation. For our simulator, we envision using a single superconducting transmon qubit $[51,52]$ for the read-out of the phonon density profile in the nanoresonator array. In this scenario, the nanoresonators would be metallized and coupled capacitively [52] to the transmon as shown in Figure 8(a). It is important that the location of the nanoresonators be determined. It can be done by mapping the nanoresonator frequencies in the array, which could be compiled either before or after the measurements using electron beam imaging (to determine with high precision the nanoresonators' geometries) and finite element simulations. Through a filtered circuit connection [53], a DC voltage V could be applied to establish the interaction between each individual nanoresonator and the transmon, which can be modeled using the Jaynes-Cummings Hamiltonian $[1,54]$ - note the same applied voltage would serve to couple the nanoresonators to each other. The full measurement Hamiltonian, incorporating the entire array of nanoresonators, would thus be given by

$$
\tilde{\mathcal{H}}=\frac{1}{2} \hbar v_{a} \sigma_{z}+\hbar \sum_{j} \omega_{j} a_{j}^{\dagger} a_{j}+\hbar \sigma_{-} \sum_{j} \lambda_{j} a_{j}^{\dagger}+\hbar \sigma_{+} \sum_{j} \lambda_{j} a_{j},
$$

where $v_{a}$ is the transmon's lowest transition energy, $\lambda_{j}$ is qubit-resonator coupling strength, $\sigma_{z}$ is the Pauli $Z$ matrix and $\sigma_{+}\left(\sigma_{-}\right)$are the qubit raising (lowering) operator. 

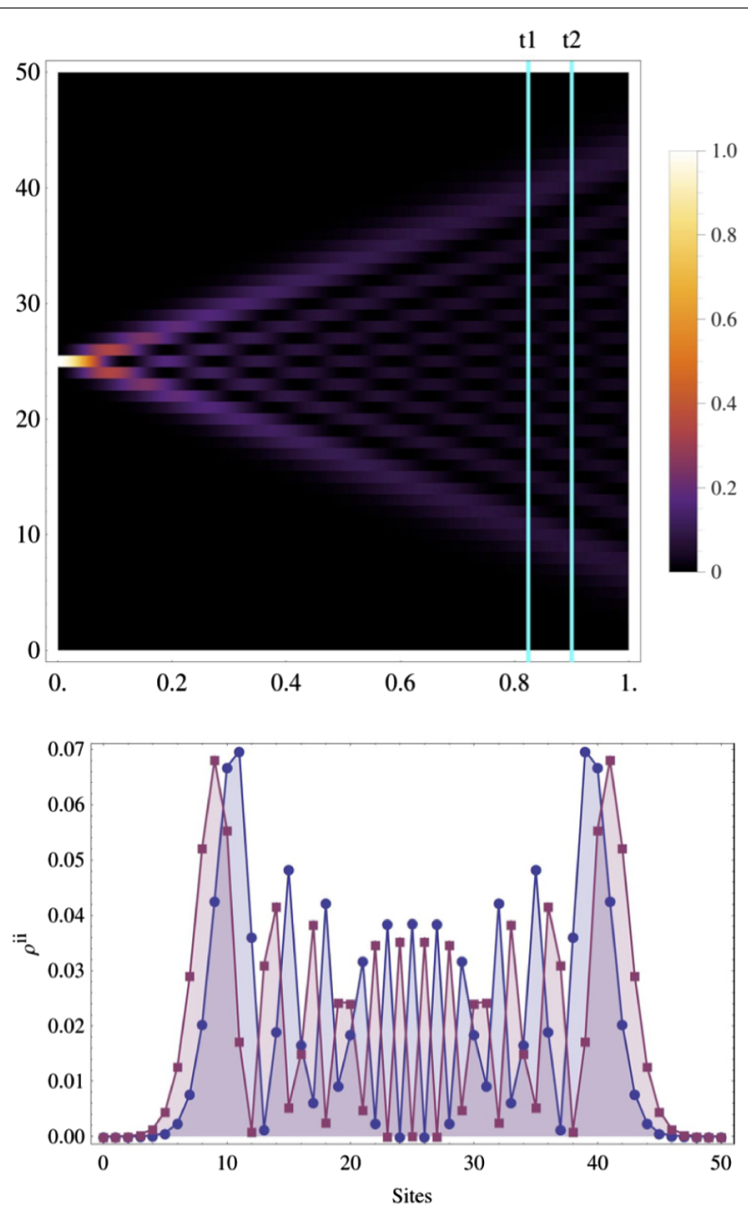

Figure 7 Phonon density probability. Phonon density probability and density profile for two specific times The profile show the typical behaviour of the continuous quantum walk, which is implemented from the Anderson Hamiltonian. Parameters are the same as in the previous figures.

The preparation and measurement protocol (Figure 8(b)) would rely upon utilizing the resonant limit of this model. To proceed, the transmon would initially be detuned in energy from all of the modes in the array and prepared in its first excited state with a microwave pi-pulse applied through a coplanar waveguide (CPW) cavity [55]. Through the application of a flux pulse, the transmon would then be brought into resonance with one of the nanoresonators and allowed to interact for one-half a Rabi cycle $\left(t_{\mathrm{Rabi}}=\pi / 2 \lambda_{j}\right)$, thus transferring its excitation to the nanoresonator mode. Next, after a predetermined delay, the location of the phonon in the nanoresonator array would be measured by scanning the transmon's transition energy $\hbar v_{a}$ (via a flux ramp) through the range of nanoresonator energies. Upon achieving resonance with the populated nanoresonator mode, the transmon would transition through a Rabi transfer back to its first excited state, which could be measured through dispersive read-out of the transmon via the CPW cavity $[55,56]$. As me mentioned the precise location of the nanoresonator could be determined from a map of frequencies using electron beam imaging and finite element simulations. Also, it should be noted that the coherent exchange of excitations through the resonant interaction between a piezoelectric disk resonator and superconducting phase qubit has been 


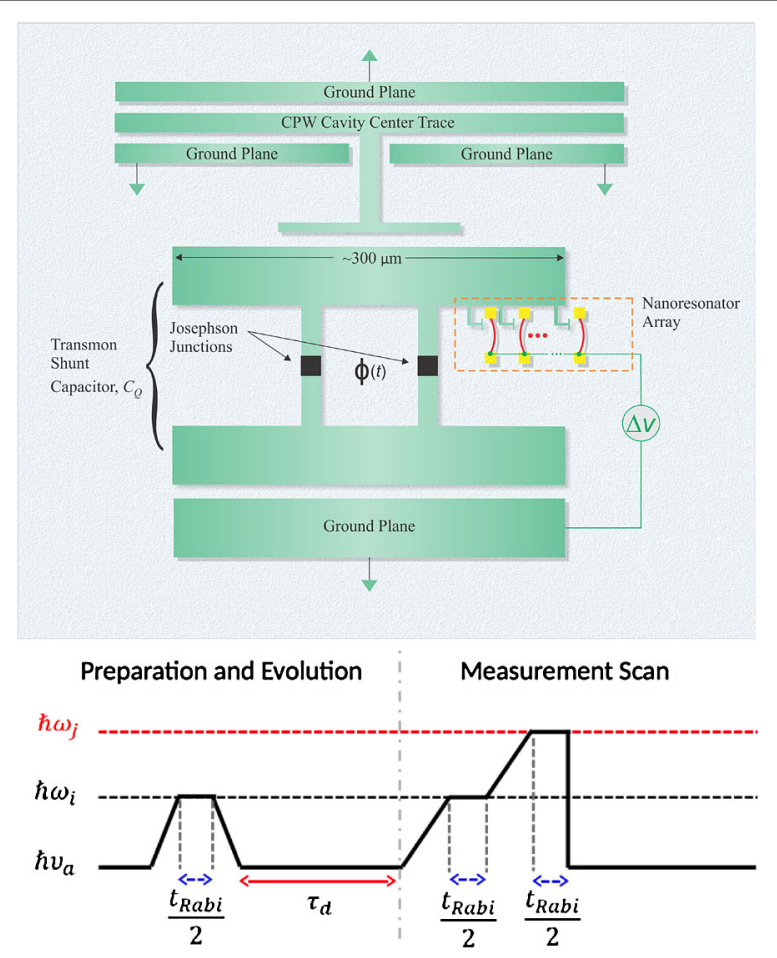

Figure 8 Illustration of the proposed layout and experimental protocol for implementing the simulation of the Anderson Hamiltonian. (a) Sample layout. The voltage-biased nanoresonator array would be capacitively-coupled to one pad of transmon's shunt capacitor $C_{Q}$. The transmon would also be capacitively-coupled to a CPW cavity for read-out. Note that layout is not drawn to scale - an array of 40 nanoresonators would extend only several microns along the transmon pad. (b) Protocol for preparing an initial photon excitation in a nanoresonator $\left(\omega_{i}\right)$ and detecting the phonon after delay time $\tau_{d}$ in a second nanoresonator $\left(\omega_{j}\right)$. The transmon transition energy $v_{a}$ would be controlled by application of flux ramp $\Phi(t)$ to the transmon. Note the ramp rates as shown are not drawn to scale.

demonstrated previously [1]; thus it is expected that a similar technique could be adapted to the case considered here.

For realization of the protocol, and to insure low thermal occupation $\left(\bar{n}<10^{-1}\right)$ of the nanoresonator modes at dilution refrigerator temperatures, it would be necessary for the nanoresonator array to be composed of ultra-high frequency (UHF) modes in the $\mathrm{GHz}$ regime [57]. With proper design of the nanostructures' geometries, the third-order, inplane flexural modes could be engineered to have frequencies varying over the range of 2 to $4 \mathrm{GHz}$ - note that varying levels of disorder $\Delta$ could be programmed into the array in a controlled manner by deliberating varying the dimensions of the nanostructures. We remark that the larger the disorder the smaller will the required array for the observation of localization. However that cannot be freely varied, since a large disorder could mean that the resonators are far from resonance to each other. That would imply the necessity to consider the counterrotating terms in Hamiltonian (3). Therefore is good to keep the limit $J \ll \Delta \ll \overline{\omega_{j}}$ for the envisaged platform.

We estimate that transmon-nanoresonator coupling strengths $\lambda_{j} \geq 1 \mathrm{MHz}$ can be achieved for this configuration by designing a tightly packed array that minimizes the spacing $d$ between nanoresonators (Figure 1). Table 1 provides estimates of $\lambda_{j}$ (and $J$ ) for geometrical parameters of the array that are realizable using standard nanolithographic techniques. With $\lambda / 2 \pi \geq 1 \mathrm{MHz}$, Rabi transfers between the transmon and nanoresonators 
Table 1 Estimates of $\omega$ assuming the standard expression from thin-beam theory for the third in-plane flexural frequency with clamped-clamped boundary conditions

\begin{tabular}{llll}
\hline $\boldsymbol{\omega} / \mathbf{2} \boldsymbol{\pi}(\mathbf{G H z})$ & $\boldsymbol{\Delta} \boldsymbol{V}(\mathbf{V})$ & $\boldsymbol{\lambda} / \mathbf{2} \boldsymbol{\pi}(\mathbf{M H z})$ & $\boldsymbol{J / 2} \boldsymbol{( \mathbf { M H z } )}$ \\
\hline 2.5 & 10 & 1.2 & 0.7 \\
2.5 & 20 & 2.3 & 2.7 \\
3.5 & 10 & 1.2 & 0.6 \\
3.5 & 20 & 2.5 & 2.3 \\
\hline
\end{tabular}

The nanoresonator is assumed to be aluminum, with the following dimensions: length $L=0.70 \mu \mathrm{m}(0.6 \mu \mathrm{m})$ for the $2.5 \mathrm{GHz}$ $(3.5 \mathrm{GHz}$ ) mode; width $w=45 \mathrm{~nm}$; thickness $t=50 \mathrm{~nm}$; The transmon-NR coupling $\lambda$ was calculated from circuit theory, assuming the systems to be in resonance, and is given by $\lambda=\omega \sqrt{\frac{d C}{d x} \frac{C \Delta V^{2}}{m \omega^{2} d C_{Q}}}$. Here $C=20 a F, d C / d x=6 \times 10^{-11} \mathrm{~F} / \mathrm{m}$ are the coupling capacitance and its first derivative respectively, which are estimated from finite element simulations assuming a gap of $d=20 \mathrm{~nm} . C_{Q}=50 \mathrm{fF}$ is the transmon shunt capacitance, which would yield a charging energy of $E_{C} / \mathrm{h}=400 \mathrm{MHz}$. Finally, $m=0.52$. $\rho$ wtL is the effective mass of the third mode, where $\rho$ is the density of aluminum. The same parameter values are assumed for the calculation of $J$.

would occur over a time-scale of at most 250 ns, which would set the minimum dwell time for each step in the flux scan to determine which nanoresonator mode is populated. For an array size of 50 nanoresonators, this would yield a maximum scan time of 25 microseconds, which in turn would set the minimum tolerable nanoresonator relaxation time. For GHz-range modes, this would require quality factors in excess of 100,000, which has been achieved with aluminum [10] and carbon-nanotube-based resonators [58] at milliKelvin temperatures, albeit at much lower resonance frequencies (10s MHz).

\section{Conclusions and perspectives}

In this paper, we have devised a quantum simulator based on nanoelectromechanical systems, for analyzing the many-body effects in quantum systems. Actually, a onedimensional array of electrostatically coupled nanoresonators is suggested to simulate the Anderson Hamiltonian. A method is present for coupling the nanoresonator electrostatically, but other means could also be explored to establish larger coupling (such as mechanical links). By introducing a controlled source of disorder to the system, we studied the localization phenomena in an array of 50 resonators. Accordingly, the population of the first excited state of a given resonator was analyzed, however, due to the discrete nature of our system, one could not expect a smooth Gaussian-to-exponential transition in the population profile.

We also studied the influence of thermal effects due to a surrounding environment, arising in real implementation of the system. By coupling the system to bosonic thermal baths, we studied the interplay between disorder and thermalization. For sufficiently low temperatures, so that the localization is experimentally detectable with the proposed simulator, the loss of phonons due to the dissipation does not immediately destroy the phonon population localization. For higher temperatures the localization is affected by the thermal pumping of excitations into the array, which generates a fully thermalized state.

Initializing the system in a known state and measuring the evolved system, effectively, are important steps in realizing a quantum simulator. Having coupled the chain of resonators to a superconducting transmon qubit, we have suggested detailed protocols to initialize and measure the system. By applying a flux pulse, the transmon qubit is brought into resonance with the desired nanoresonator which allows to interact with the resonator hence transferring its excitation to the nanoresonator. After system evolution, the location of the phonon in the nanoresonator array can be measured by scanning the transmon's 
transition energy through the range of nanoresonator energies. Having achieved resonance with the populated nanoresonator, the transmon would transfer back to its first excited state. The transmon can then be measured through dispersive read-out via the CPW cavity.

Beside simulating Anderson localization, we have also discussed the possibility of using the proposed quantum simulator for implementing the continuous-time quantum walk dynamics. The system allows to realize localization and decoherence in continuous-time quantum walks. The initialization and measurement protocols permit to inject several phonons in the chain to investigate the multi-particle quantum walks. A (non-trivial) two-dimensional version of the suggested simulator can be used to implement twodimensional quantum walks. In such case, quantum algorithms can be implemented. It is worth to mention that to some extent the present discussion applies as well to optomechanical systems, such as in Refs. [59, 60], whose architectures are worth to be explored for quantum simulation purposes. Moreover, given the ability to control individual resonator excitations by scanning the transmon frequency, it is possible to extend the present investigation to the situation including on-site interactions. When several realizations are taken into account the effective Hamiltonian averages out to (4) plus on-site interactions on all sites, in a similar way to the Bose-Hubbard model, allowing investigation of the allowed phases and respective quantum phase transitions when the parameters are varied $[61,62]$. In that way this would allow for investigation nonequilibrium steady states of quantum many-body models in similar fashion to other proposed simulators [63, 64].

Competing interests

The authors declare that they have no competing interests.

Authors' contributions

All Authors have contributed equally to the manuscript.

Author details

${ }^{1}$ Instituto de Física "Gleb Wataghin", Universidade Estadual de Campinas, Campinas, SP 13083-970, Brazil. ${ }^{2}$ Coordenação de Ciência da Computação, Universidade Estadual do Piauí (UESPI), Parnaíba, PI 64202-220, Brazil. ${ }^{3}$ Department of Physics, Syracuse University, Syracuse, NY 13244-1130, USA.

\section{Acknowledgements}

JKM acknowledges financial support from Brazilian National Council for Scientific and Technological Development (CNPq), grant PDJ 165941/2014-6. MCO acknowledges support by FAPESP and CNPq through the National Institute for Science and Technology on Quantum Information and the Research Center in Optics and Photonics (CePOF). MDL acknowledges support for this work provided by the National Science Foundation under Grant DMR-1056423 and Grant DMR-1312421.

d Discrete models bring essentially the same qualitative results as continuous ones regarding localization effects, and they are solvable for many disorder distributions.

e In this work all the simulations were performed for 500 realizations of the disorder
}

Received: 16 December 2015 Accepted: 30 May 2016 Published online: 08 June 2016

\section{References}

1. O'Connell AD, Hofheinz M, Ansmann M, Bialczak RC, Lenander M, Lucero E, Neeley M, Sank D, Wang H, Weides M, Wenner J, Martinis JM, Cleland AN. Quantum ground state and single-phonon control of a mechanical resonator. Nature. 2010;464(7289):697-703.

2. Teufel JD, Donner T, Li D, Harlow JW, Allman MS, Cicak K, Sirois AJ, Whittaker JD, Lehnert KW, Simmonds RW. Sideband cooling of micromechanical motion to the quantum ground state. Nature. 2011;475(7356):359-63. 
3. Chan J, Alegre TPM, Safavi-Naeini AH, Hill JT, Krause A, Groblacher S, Aspelmeyer M, Painter O. Laser cooling of a nanomechanical oscillator into its quantum ground state. Nature. 2011;478(7367):89-92.

4. Wollman EE, Lei CU, Weinstein AJ, Suh J, Kronwald A, Marquardt F, Clerk AA, Schwab KC. Quantum squeezing of motion in a mechanical resonator. Science. 2015;349(6251):952-5.

http://science.sciencemag.org/content/349/6251/952. ISSN 0036-8075. doi:10.1126/science.aac5138.

5. Lecocq F, Clark JB, Simmonds RW, Aumentado J, Teufel JD. Quantum nondemolition measurement of a nonclassical state of a massive object. Phys Rev X. 2015;5:041037. http://link.aps.org/doi/10.1103/PhysRevX.5.041037. doi:10.1103/PhysRevX.5.041037.

6. Pirkkalainen J-M, Damskägg E, Brandt M, Massel F, Sillanpää MA. Squeezing of quantum noise of motion in a micromechanical resonator. Phys Rev Lett. 2015;115:243601. http://link.aps.org/doi/10.1103/PhysRevLett.115.243601. doi:10.1103/PhysRevLett.115.243601.

7. Wilson DJ, Sudhir V, Piro N, Schilling R, Ghadimi A, Kippenberg TJ. Measurement-based control of a mechanical oscillator at its thermal decoherence rate. Nature. 2015;524(7565):325-9.

8. Milburn GJ, Woolley MJ. An introduction to quantum optomechanics. Acta Phys Slovaca. 2011;61(5):483-601.

9. Suh J, Weinstein AJ, Lei CU, Wollman EE, Steinke SK, Meystre P, Clerk AA, Schwab KC. Mechanically detecting and avoiding the quantum fluctuations of a microwave field. Science. 2014;344(6189):1262-5.

10. Palomaki TA, Teufel JD, Simmonds RW, Lehnert KW. Entangling mechanical motion with microwave fields. Science. 2013:342(6159):710-3.

11. Lecocq F, Teufel JD, Aumentado J, Simmonds RW. Resolving the vacuum fluctuations of an optomechanical system using an artificial atom. Nat Phys. 2015; doi:10.1038/nphys3365.

12. Georgescu IM, Ashhab S, Nori F. Quantum simulation. Rev Mod Phys. 2014;86:153-85. http://ink.aps.org/doi/10.1103/RevModPhys.86.153. doi:10.1103/RevModPhys.86.153.

13. Buluta I, Nori F. Quantum simulators. Science. 2009;326(5949):108-11.

14. Bloch I, Dalibard J, Nascimbène S. Quantum simulations with ultracold quantum gases. Nat Phys. 2012;8(4):267-76.

15. Blatt R, Roos CF. Quantum simulations with trapped ions. Nat Phys. 2012;8(4):277-84.

16. Aspuru-Guzik A, Walther P. Photonic quantum simulators. Nat Phys. 2012;8(4):285-91

17. Houck AA, Türeci HE, Koch J. On-chip quantum simulation with superconducting circuits. Nat Phys. 2012;8(4):292-9.

18. Schmidt S, Koch J. Circuit QED lattices: towards quantum simulation with superconducting circuits. Ann Phys. 2013;525(6):395-412.

19. Ludwig M, Marquardt F. Quantum many-body dynamics in optomechanical arrays. Phys Rev Lett. 2013;111:073603. http://link.aps.org/doi/10.1103/PhysRevLett.111.073603. doi:10.1103/PhysRevLett.111.073603.

20. Anderson PW. Absence of diffusion in certain random lattices. Phys Rev. 1958;109:1492-505. http://link.aps.org/doi/10.1103/PhysRev.109.1492. doi:10.1103/PhysRev.109.1492.

21. Lee PA, Ramakrishnan TV. Disordered electronic systems. Rev Mod Phys. 1985;57:287-337. http://link.aps.org/doi/10.1103/RevModPhys.57.287. doi:10.1103/RevModPhys.57.287.

22. Kramer B, MacKinnon A. Localization: theory and experiment. Rep Prog Phys. 1993;56(12):1469.

23. Dalichaouch R, Armstrong JP, Schultz S, Platzman PM, McCall SL. Microwave localization by two-dimensional random scattering. Nature. 1991;354(6348):53-5.

24. Chabanov AA, Stoytchev M, Genack AZ. Statistical signatures of photon localization. Nature. 2000;404(6780):850-3.

25. Chabanov AA, Zhang ZQ, Genack AZ. Breakdown of diffusion in dynamics of extended waves in mesoscopic media. Phys Rev Lett. 2003;90:203903. http://link.aps.org/doi/10.1103/PhysRevLett.90.203903. doi:10.1103/PhysRevLett.90.203903.

26. Wiersma DS, Bartolini P, Lagendijk A, Righini R. Localization of light in a disordered medium. Nature. 1997:390(6661):671-3.

27. Störzer M, Gross P, Aegerter CM, Maret G. Observation of the critical regime near Anderson localization of light. Phys Rev Lett. 2006:96:063904. http://link.aps.org/doi/10.1103/PhysRevLett.96.063904. doi:10.1 103/PhysRevLett.96.063904.

28. Aegerter CM, Störzer M, Maret G. Experimental determination of critical exponents in Anderson localisation of light. Europhys Lett. 2006;75(4):562.

29. Schwartz T, Bartal G, Fishman S, Segev M. Transport and Anderson localization in disordered two-dimensional photonic lattices. Nature. 2007;446(7131):52-5.

30. Lahini Y, Avidan A, Pozzi F, Sorel M, Morandotti R, Christodoulides DN, Silberberg Y. Anderson localization and nonlinearity in one-dimensional disordered photonic lattices. Phys Rev Lett. 2008;100:013906. http://link.aps.org/doi/10.1103/PhysRevLett.100.013906. doi:10.1103/PhysRevLett.100.013906.

31. Sperling T, Buehrer W, Aegerter CM, Maret G. Direct determination of the transition to localization of light in three dimensions. Nat Photonics. 2013;7(1):48-52.

32. Segev M, Silberberg Y, Christodoulides DN. Anderson localization of light. Nat Photonics. 2013;7(3):197-204.

33. Weaver RL. Anderson localization of ultrasound. Wave Motion. 1990;12(2):129-42.

34. Hu H, Strybulevych A, Page JH, Skipetrov SE, van Tiggelen BA. Localization of ultrasound in a three-dimensional elastic network. Nat Phys. 2008;4(12):945-8.

35. Billy J, Josse V, Zuo Z, Bernard A, Hambrecht B, Lugan P, Clément D, Sanchez-Palencia L, Bouyer P, Aspect A. Direct observation of Anderson localization of matter waves in a controlled disorder. Nature. 2008;453(7197):891-4.

36. Roati G, D'Errico C, Fallani L, Fattori M, Fort C, Zaccanti M, Modugno G, Modugno M, Inguscio M. Anderson localization of a non-interacting Bose-Einstein condensate. Nature. 2008:453(7197):895-8.

37. Chabé J, Lemarié G, Grémaud B, Delande D, Szriftgiser P, Garreau JC. Experimental observation of the Anderson metal-insulator transition with atomic matter waves. Phys Rev Lett. 2008;101:255702. http://link.aps.org/doi/10.1103/PhysRevLett.101.255702. doi:10.1103/PhysRevLett.101.255702.

38. Kondov SS, McGehee WR, Zirbel JJ, DeMarco B. Three-dimensional Anderson localization of ultracold matter. Science. 2011;334(6052):66-8.

39. Jendrzejewski F, Bernard A, Mueller K, Cheinet P, Josse V, Piraud M, Pezzé L, Sanchez-Palencia L, Aspect A, Bouyer P. Three-dimensional localization of ultracold atoms in an optical disordered potential. Nat Phys. 2012;8(5):398-403.

40. McGehee WR, Kondov SS, Xu W, Zirbel JJ, DeMarco B. Three-dimensional Anderson localization in variable scale disorder. Phys Rev Lett. 2013;111:145303. http://link.aps.org/doi/10.1103/PhysRevLett.111.145303. doi:10.1103/PhysRevLett.111.145303. 
41. Farhi E, Gutmann S. Quantum computation and decision trees. Phys Rev A. 1998;58(2):915.

42. Portugal R. Quantum walks and search algorithms. New York, NY: Springer; 2013. http://link.springer.com/10.1007/978-1-4614-6336-8. ISBN 978-1-4614-6335-1. doi:10.1007/978-1-4614-6336-8.

43. Du J, Li H, Xu X, Shi M, Wu J, Zhou X, Han R. Experimental implementation of the quantum random-walk algorithm. Phys Rev A. 2003;67:042316.

44. Perets HB, Lahini Y, Pozzi F, Sorel M, Morandotti R, Silberberg Y. Realization of quantum walks with negligible decoherence in waveguide lattices. Phys Rev Lett. 2008;100(17):170506.

45. Childs AM. Universal computation by quantum walk. Phys Rev Lett. 2009;102:180501. http://link.aps.org/doi/10.1103/PhysRevLett.102.180501. doi:10.1103/PhysRevLett.102.180501.

46. Aspelmeyer M, Kippenberg TJ, Marquardt F. Cavity optomechanics. Rev Mod Phys. 2014;86:1391-452. http://link.aps.org/doi/10.1103/RevModPhys.86.1391. doi:10.1103/RevModPhys.86.1391.

47. Abrahams E, Anderson PW, Licciardello DC, Ramakrishnan TV. Scaling theory of localization: absence of quantum diffusion in two dimensions. Phys Rev Lett. 1979;42:673-6.

48. Müller CA, Delande D. Ultracold gases and quantum information. In: Miniatura C et al., editors. Lecture notes of the les houches summer school in Singapore, vol. 91. 2009.

49. Wootters WK. Entanglement of formation of an arbitrary state of two qubits. Phys Rev Lett. 1998;80(10):2245-8.

50. Almeida MP, de Melo F, Hor-Meyll M, Salles A, Walborn SP, Ribeiro PHS, Davidovich L. Environment-induced sudden death of entanglement. Science. 2007;316(5824):579-82.

51. Koch J, Terri MY, Gambetta J, Houck AA, Schuster DI, Majer J, Blais A, Devoret MH, Girvin SM, Schoelkopf RJ. Charge-insensitive qubit design derived from the cooper pair box. Phys Rev A. 2007;76(4):042319.

52. Pirkkalainen J-M, Cho SU, Li J, Paraoanu GS, Hakonen PJ, Sillanpää MA. Hybrid circuit cavity quantum electrodynamics with a micromechanical resonator. Nature. 2013;494(7436):211-5.

53. Hao Y, Rouxinol F, LaHaye MD. Development of a broadband reflective T-filter for voltage biasing high-q superconducting microwave cavities. Appl Phys Lett. 2014:105(22):222603.

54. Irish EK, Schwab K. Quantum measurement of a coupled nanomechanical resonator cooper-pair box system. Phys Rev B. 2003:68:155311. http://link.aps.org/doi/10.1103/PhysRevB.68.155311. doi:10.1103/PhysRevB.68.155311.

55. Blais A, Huang R-S, Wallraff A, Girvin SM, Schoelkopf RJ. Cavity quantum electrodynamics for superconducting electrical circuits: an architecture for quantum computation. Phys Rev A. 2004;69(6):062320.

56. Wallraff A, Schuster DI, Blais A, Frunzio L, Majer J, Devoret MH, Girvin SM, Schoelkopf RJ. Approaching unit visibility for control of a superconducting qubit with dispersive readout. Phys Rev Lett. 2005;95(6):060501.

57. Huang XMH, Zorman CA, Mehregany M, Roukes ML. Nanoelectromechanical systems: nanodevice motion at microwave frequencies. Nature. 2003;421(6922):496

58. Moser J, Eichler A, Güttinger J, Dykman Ml, Bachtold A. Nanotube mechanical resonators with quality factors of up to 5 million. Nat Nanotechnol. 2014;9(12):1007-11.

59. Midtvedt D, Isacsson A, Croy A. Nonlinear phononics using atomically thin membranes. Nat Commun. 2014; 5 . http://dx.doi.org/10.1038/ncomms5838

60. Xuereb A, Genes C, Pupillo G, Paternostro M, Dantan A. Reconfigurable long-range phonon dynamics in optomechanical arrays. Phys Rev Lett. 2014;112:133604. http://link.aps.org/doi/10.1103/PhysRevLett.112.133604. doi:10.1103/PhysRevLett.112.133604.

61. de Oliveira MC, da Cunha BR. Collision-dependent atom tunnelling rate: Bose-Einstein condensates in double and multiple well traps. Int J Mod Phys B. 2009;23(32):5867-80.

62. Farias RJC, de Oliveira MC. Entanglement and the Mott insulator-superfluid phase transition in bosonic atom chains. J Phys Condens Matter. 2010;22(24):245603. http://stacks.iop.org/0953-8984/22/i=24/a=245603.

63. Grujic T, Clark SR, Jaksch D, Angelakis DG. Non-equilibrium many-body effects in driven nonlinear resonator arrays. New J Phys. 2012;14(10):103025. http://stacks.iop.org/1367-2630/14/i=10/a=103025.

64. Schmidt S, Koch J. Circuit QED lattices: towards quantum simulation with superconducting circuits. Annalen der Physik. 2013;525(6):395-412. http://dx.doi.org/10.1002/andp.201200261. ISSN 1521-3889. doi:10.1002/andp.201200261.

65. van Tiggelen BA. Anderson localization of waves in diffuse waves in complex media (ed. J.-P. fouque). The Netherlands: Kluwer Academic Publisher; 1999.

66. Izrailev FM, Krokhin AA. Localization and the mobility edge in one-dimensional potentials with correlated disorder. Phys Rev Lett. 1999;82:4062-5.

\section{Submit your manuscript to a SpringerOpen ${ }^{\circ}$ journal and benefit from:}

- Convenient online submission

- Rigorous peer review

Immediate publication on acceptance

- Open access: articles freely available online

- High visibility within the field

- Retaining the copyright to your article

Submit your next manuscript at $\boldsymbol{s p r i n g e r o p e n . c o m ~}$ 